\title{
High-energy spatiotemporal solitons in GRIN fiber
}

\author{
F. Mangini', M. Zitelli², M. Ferraro², D.S. Kharenko³, A. Niang1, A. Tonello4, V. Couderc ${ }^{4}$, S. Wabnitz ${ }^{2,3}$ \\ 1. Department of Information Engineering (DII), University of Brescia, Via Branze 38, 25123 Brescia, Italy. \\ 2. Department of Information Engineering, Electronics and Telecommunications (DIET), \\ Sapienza University of Rome, Via Eudossiana 18, 00184 Rome, Italy. \\ 3. Novosibirsk State University, Pirogova 1, Novosibirsk 630090, Russia. \\ 4. Université de Limoges, XLIM, UMR CNRS 7252, 123 Avenue A. Thomas, 87060 Limoges, France
}

In the last decade, the study of the propagation of ultrashort pulses in multimode (MM) fibers has seen a renewed interest, thanks to the potential that this technology can offer for guided optical communications [1-3]. Despite numerous studies in the literature, several aspects are still to be fully understood. In this work, we consider the high-energy regime, up to reaching the fiber damage threshold, of spatiotemporal soliton generation in MM fibers. In particular, we study the propagation of femtosecond pulses in a MM fiber with a graded-index (GRIN) core profile. Intrapulse Raman scattering leads to the fission of the initial pulse into many MM solitons, which undergo Raman self-frequency shift (SSFS) [4].

In the present study, we reveal the presence of a new type of nonlinear propagation regime in MM fibers, where stable spatiotemporal solitons are created by the fission of the initial pulse. These solitons have different amplitudes and wavelengths, but nearly equal time duration. To study the generation and fission of high-energy MM solitons in the anomalous dispersion regime, we used a hybrid optical parametric amplifier (OPA) generating pulses with hyperbolic secant temporal profile of 130-70 fs duration at $1550 \mathrm{~nm}$, and repetition rate of $33 \mathrm{kHz}$. The input pulse energy was varied between $0.1 \mathrm{~nJ}$ and $500 \mathrm{~nJ}$ by a variable attenuator. The laser beam was coupled with a $30 \mathrm{~cm}$ long multimode standard 50/125 GRIN fiber with relative index difference $\Delta=0.0103$ (cladding refractive index is 1.457 ) and chromatic dispersion $\beta_{2}=-22 \mathrm{ps}^{2} / \mathrm{km}$ by a $50 \mathrm{~mm}$ lens producing a $30 \mu \mathrm{m}$ beam waist at the fiber input. Numerical simulations were also conducted to reproduce the phenomenon of fission using an exact 3D+1 vector model that includes second, third, and fourth-order dispersion, Kerr, and Raman nonlinearities. We neglected the contribution of polarization mode dispersion, since it is negligible for the fiber lengths involved in our experiments. We included a phenomenological two-photon absorption (TPA) term, to take into account the presence of nonlinear losses (TPA coefficient is taken to be $\alpha_{2}=10^{-16} \mathrm{~m} / \mathrm{W}$ by fitting the experimental SSFS values). Random phase noise was added to the input field, to describe the generation of intensity speckles in the output transverse field, and to seed the generated dispersive waves.

In Fig.1.a the output spectrum is shown for different values of the input pulse energy. It shows the creation of several Raman solitons. In particular, one can observe how the Raman-induced SSFS tends to saturate for energies higher than $200 \mathrm{~nJ}$. Correspondingly, the total output energy is clamped, as shown in Fig. 1.b : we revealed that this is due to the presence of high (up to $80 \%$ transmission drop) nonlinear loss in the first few cm of MM fiber. We ascribe the nonlinear loss to multiphoton absorption by defects of the Germanium doped fiber, which is enhanced at the points of peak intensity, owing to beam self-imaging. Therefore, two distinct propagation regimes exist: in the first, only weak linear losses are present ; in the second, the output energy remains clamped to a nearly constant value. From Fig.1.b, we can see that 100-150 nJ is the input energy boundary between these two regimes. Fig. 1.c shows that the soliton pulsewidth remains nearly constant (50-60 fs) as the soliton energy grows larger than about 10-15 nJ. Remarkably, in the nonlinear loss regime nearly all of the transmitted energy is funneled into high-energy spatiotemporal soliton pulses with a bell-shaped, high-quality transverse beam profile.
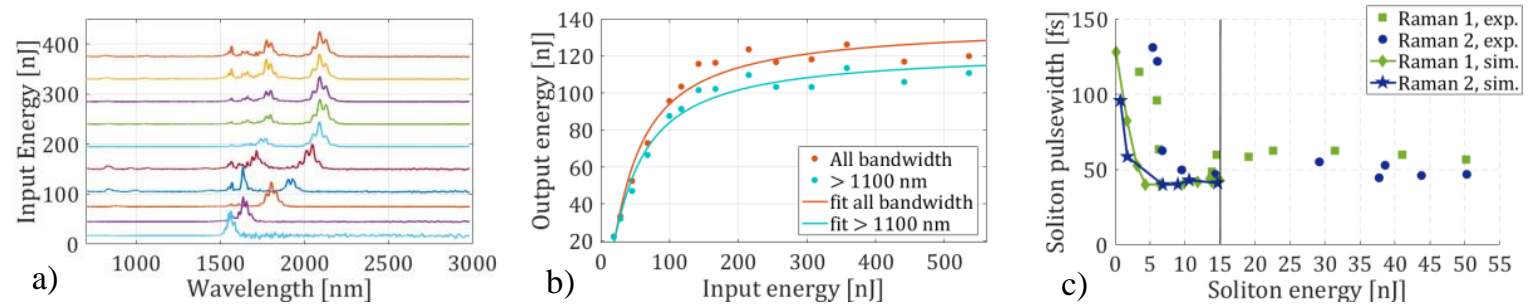

Fig. 1 a) Output spectrum evolution at different output energy (linear scale); b) experimental output energy vs. input energy, in the overall bandwidth and for wavelength above $1100 \mathrm{~nm}$; c) Soliton pulsewidth vs. soliton energy.

This work was supported by the European Research Council (grant No. 740355), and by the Russian Ministry of Science and Education, (grant No. 14.Y26.31.0017).

\section{References}

[1] W. H. Renninger et al., "Optical solitons in graded-index multimode fibres," Nat. Commun. 4, 1719 (2013).

[2] L. G. Wright, et al., "Spatiotemporal dynamics of multimode optical solitons," Opt. Express 23, 3492 (2015).

[3] K. Krupa, et al., "Multimode nonlinear fiber optics, a spatiotemporal avenue," APL Photonics 11, 110901 (2019).

[4] A.S. Ashan and G.P. Agrawal, "Spatio-temporal enhancement of Raman-induced frequency shift in graded-index multimode fibers," Opt.

Lett. 44, 2637 (2019) 